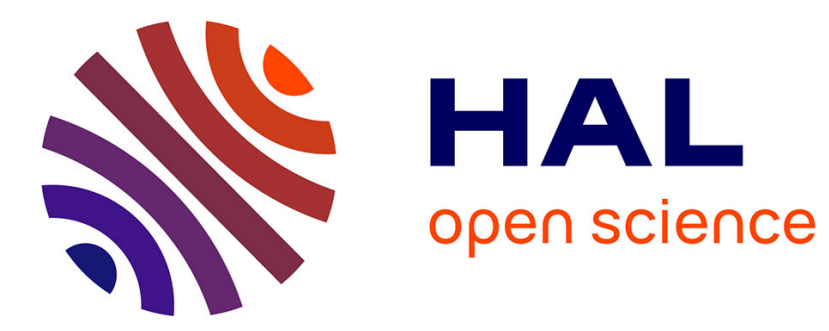

\title{
Appareillage automatique pour les mesures de résistivité et de constante de Hall
}

A. Gouskov

\section{To cite this version:}

A. Gouskov. Appareillage automatique pour les mesures de résistivité et de constante de Hall. Revue de Physique Appliquée, 1969, 4 (4), pp.491-494. 10.1051/rphysap:0196900404049100 . jpa-00243317

\section{HAL Id: jpa-00243317 https://hal.science/jpa-00243317}

Submitted on 1 Jan 1969

HAL is a multi-disciplinary open access archive for the deposit and dissemination of scientific research documents, whether they are published or not. The documents may come from teaching and research institutions in France or abroad, or from public or private research centers.
L'archive ouverte pluridisciplinaire HAL, est destinée au dépôt et à la diffusion de documents scientifiques de niveau recherche, publiés ou non, émanant des établissements d'enseignement et de recherche français ou étrangers, des laboratoires publics ou privés. 


\title{
APPAREILLAGE AUTOMATIQUE POUR LES MESURES DE RÉSISTIVITÉ ET DE CONSTANTE DE HALL
}

\author{
Par A. GOUSKOV, \\ Centre d'Études d'Électronique des Solides de Montpellier (associé au C.N.R.S.). \\ (Reçu le 15 juillet 1969.)
}

\begin{abstract}
Résumé. - On décrit un appareillage automatique destiné aux mesures de résistivité et d'effet Hall en fonction de la température.

Les données s'inscrivent sur le papier d'une machine à écrire ou directement sur cartes perforées, ce qui permet d'éviter les calculs intermédiaires et d'avoir ainsi directement les valeurs de la résistivité et de la constante de Hall des échantillons en fonction de la température.

L'appareillage permet d'étudier simultanément quatre échantillons.

Les tests ont essentiellement porté sur des échantillons de GaSb diversement dopés.
\end{abstract}

\begin{abstract}
An automatic device for resistivity and Hall effect measurements as a function of temperature is described.

The output data can be transcribed on an electric typewriter or it can be punched on I.B.M. cards. In either form the values of the resistivity and the Hall coefficient as a function of temperature are presented. This allows us to avoid the intermediate calculations.

Four samples can be studied simultaneously with this device.

The tested samples were GaSb with various dopings.
\end{abstract}

I. Introduction. - La plupart des études faites sur un semiconducteur nécessitent la connaissance préalable de ses caractéristiques, et en particulier de sa résistivité et de sa constante de Hall à différentes températures. Ce travail de routine a nécessité la réalisation d'un dispositif automatique de mesures capable d'étudier quatre échantillons simultanément.

L'appareil effectue les mesures sur des échantillons d'épaisseur constante en forme de croix ou de trèfle à quatre feuilles. Une machine à écrire inscrit toutes les mesures permettant les calculs de résistivité et de constante de Hall par la méthode de Van der Pauw [1], mais on peut également utiliser une perforatrice de cartes. Cette dernière utilisation évite les calculs intermédiaires qui sont confiés à un ordinateur et aboutit à l'obtention quasi instantanée des valeurs de la résistivité $\rho$ et de la constante de Hall $R_{\mathrm{H}}$ en fonction de la température.

II. Principe de la mesure - Nous reprenons ici les résultats énoncés par Van der Pauw.

Plaçons-nous dans le cas favorable où l'échantillon à mesurer a une épaisseur constante $\mathrm{d}$ et présente l'aspect d'un trèfle à quatre feuilles, les contacts électriques étant suffisamment petits, situés sur la tranche, et au milieu de chaque feuille.

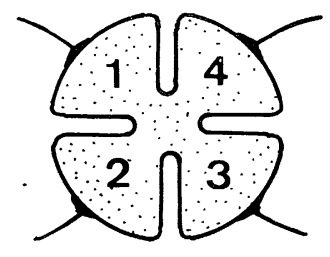

Numérotons de 1 à 4 les quatre branches :

1. Mesure de $\rho$. - Soit $i_{12}$ un courant amené en 1 et recueilli en 2 .

Soit $v_{34}$ la tension mesurée entre 3 et 4 . Définissons :

$$
R_{1234}=\frac{v_{34}}{i_{12}} .
$$

Soit $i_{14}$ un courant amené de 1 et recueilli en 4 .

Soit $v_{23}$ la tension mesurée entre 2 et 3 . Définissons de même :

$$
R_{1423}=\frac{v_{23}}{i_{14}} .
$$

Entre ces deux résistances existe la relation :

$$
\exp \left(-\frac{\pi d}{\rho} R_{1234}\right)+\exp \left(-\frac{\pi d}{\rho} R_{1423}\right)=1
$$


qui peut être ramenée à l'expression :

$$
\rho=\frac{\pi d}{\log 2} \cdot \frac{R_{1234}+R_{1423}}{2} \cdot f
$$

dans laquelle $f$ est un facteur ne dépendant que du rapport $\frac{R_{1234}}{R_{1423}}$.

On peut prévoir qu'étant donné la géométrie de l'échantillon $\frac{R_{1234}}{R_{1423}} \# 1$, on a alors : $f=1$.

2. Mesure De $R_{\mathrm{H}}$ - Soit $i_{\mathbf{1 3}}$ un courant amené en 1 et recueilli en 3 .

Soit $v_{24}$ la tension mesurée entre 2 et 4 . Définissons :

$$
R_{1324}=\frac{v_{24}}{i_{13}}
$$

Lorsqu'on applique un champ magnétique $B$ perpendiculaire au plan de l'échantillon, la tension $v_{\mathbf{2 4}}$ change et prend la valeur $v_{24}^{\prime}$. On montre que cette variation de tension obéit à la loi :

$$
v_{24}^{\prime}-v_{24}=R_{\mathrm{H}} \cdot B \cdot \frac{i_{13}}{d}
$$

d'où :

$$
R_{\mathrm{H}}=\frac{\left(v_{24}^{\prime}-v_{24}\right) d}{B \cdot i_{13}} .
$$

III. Technique de la mesure. - L'obtention des valeurs de la résistivité $\rho$ et de la constante de Hall $R_{\mathrm{H}}$ de l'échantillon exige donc sur le plan expérimental de disposer d'un courant $i$ que l'on fait circuler entre deux des bornes de l'échantillon, la tension étant mesurée entre les deux bornes restantes.

Par mesure de précaution, afin de dépister des anomalies dues par exemple à des contacts redresseurs, les mesures sont faites chaque fois pour les deux sens de passage du courant.

Les mesures de la résistivité exigent le passage du courant entre 1 et 2 d'une part, entre 1 et 4 d'autre part. Les mesures du coefficient de Hall exigent le passage du courant entre 1 et 3 sans application du champ magnétique d'une part, avec application $\mathrm{du}$ champ magnétique d'autre part.
En outre, la température ne doit pas évoluer au cours du relevé complet de la série de mesures nécessaires au calcul de $\rho$ et de $R_{\mathrm{H}}$. Deux thermocouples $\mathrm{T}_{1}$ et $T_{2}$ placés au voisinage des échantillons indiquent la température.

Ces considérations nous ont amené à fournir à la machine un programme de mesures représenté dans le tableau I où quatre échantillons peuvent être étudiés simultanément.

Ge cycle de mesures s'inscrit sur le papier d'une machine à écrire ligne par ligne. Le temps d'inscription d'une mesure dure une seconde, ce qui impose, compte tenu des temps morts d'établissement des circuits et des marges de sécurité, l'inscription d'une ligne toutes les $15 \mathrm{~s}$ et donc d'un cycle complet toutes les $2 \mathrm{mn}$. Il ne faut pas que la température varie sensiblement dans cet intervalle de $2 \mathrm{mn}$.

L'appareil est conçu de telle sorte qu'il inscrit deux cycles complets de mesures consécutifs, puis s'arrête; il reprend ses mesures au bout d'un temps affiché sur une horloge. Ge temps est affiché par l'utilisateur en fonction des conditions de variation de la température. L'arrêt entre les deux séries de mesures est réglable entre 0 et $22 \mathrm{mn}$.

Parallèlement, les données peuvent être inscrites sur cartes perforées qui sont ensuite confiées à un ordinateur.

IV. Réalisation de l'appareillage. - Les mesures automatiques de résistivité et de constante de Hall nécessitent évidemment l'emploi des appareils habituels : cryostat à hélium, source de courant continu et électroaimant. L'appareil de mesure ne peut plus être un simple voltmètre à grande impédance, il est remplacé par une centrale de mesure Dynamco Microscan 6400 reliée à une machine à écrire imprimant les tensions mesurées.

Un organe central est destiné à coordonner le for.ctionnement de l'ensemble. Il est chargé de connecter au moyen de relais les bornes des échantillons, d'une part au générateur de courant, d'autre part à la centrale de mesure dans l'ordre imposé par le programme de travail du tableau I, puis de déclencher les mesures. Il doit également mettre en marche l'électro-

\begin{tabular}{|c|c|c|c|c|c|c|c|}
\hline & & & & ÉGH. 1 & Е́сан. 2 & Éсан. 3 & Éch. \\
\hline 1 & $\mathrm{~T}_{1}$ & $\mathrm{~T}_{2}$ & $i_{12}$ & $v_{34}$ & $v_{34}$ & $v_{34}$ & $v_{34}$ \\
\hline 2 & $\mathrm{~T}_{1}$ & $\mathrm{~T}_{2}$ & $i_{21}$ & $v_{34}$ & $v_{34}$ & $v_{34}$ & $v_{34}$ \\
\hline 3 & $\mathrm{~T}_{1}$ & $\mathrm{~T}_{2}$ & $i_{13}$ & $v_{24}$ & $v_{24}$ & $v_{24}$ & $v_{24}$ \\
\hline 4 & $\mathrm{~T}_{1}$ & $\mathrm{~T}_{2}$ & $i_{31}$ & $v_{24}$ & $v_{24}$ & $v_{24}$ & $v_{24}$ \\
\hline 5 & $\mathrm{~T}_{1}$ & $\mathrm{~T}_{2}$ & $i_{14}$ & $v_{23}$ & $v_{23}$ & $v_{23}$ & $v_{23}$ \\
\hline 6 & $T_{1}$ & $\mathrm{~T}_{2}$ & $i_{41}$ & $v_{23}$ & $v_{23}$ & $v_{23}$ & $v_{23}$ \\
\hline 7 & $\mathrm{~T}_{1}$ & $\mathrm{~T}_{2}$ & $i_{13}$ & $v_{24}^{\prime}$ & $v_{24}^{\prime}$ & $v_{24}^{\prime}$ & $v_{24}^{\prime}$ \\
\hline 8 & $\mathrm{~T}_{1}$ & $\mathrm{~T}_{2}$ & $i_{31}$ & $v_{24}^{\prime}$ & $v_{24}^{\prime}$ & $v_{24}^{\prime}$ & $v_{24}^{\prime}$ \\
\hline
\end{tabular}
aimant au moment de l'inscription des lignes 7 et 8 du tableau I.

\section{TABLEAU I}




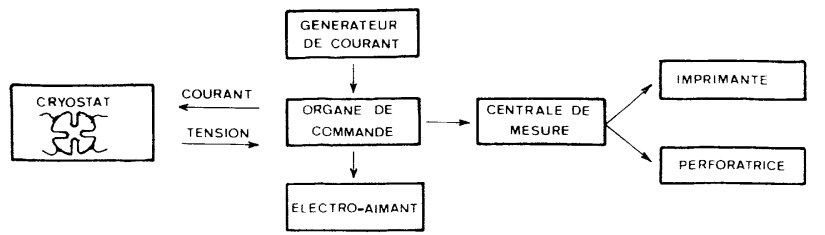

FIG. 1. - Tableau synoptique du montage.

Nous présentons (ifg. 1) un tableau synoptique de l'appareillage.

1. Le cryostat à hélium est un Sulfrian Select-AStat. La queue du cryostat est un parallélépipède de cuivre rouge de dimensions $20 \times 40 \times 10 \mathrm{~mm}$. Les deux faces les plus grandes reçoivent deux échantillons chacune. Afin d'éviter les court-circuits, une feuille de mylar de $6 \mu$ d'épaisseur est interposée entre la surface de cuivre et les échantillons. Un contact thermique suffisant est obtenu en recouvrant les surfaces avec un peu de graisse à vide.

Un collier muni de bornes aboutissant par des conducteurs à l'organe de commande enserre la queue du cryostat juste au-dessus du support d'échantillons, il permet de relier aisément les contacts des échantillons à ces bornes.

2. Le GÉNÉRATEUR DE GOURANT permet de régler le débit d'une façon continue de $0,1 \mathrm{~mA}$ à $125 \mathrm{~mA}$.

La chute de tension mesurée par la centrale de mesure aux bornes d'une résistance de $100 \Omega$ permet de connaître la valeur du courant débité (fig. 2).

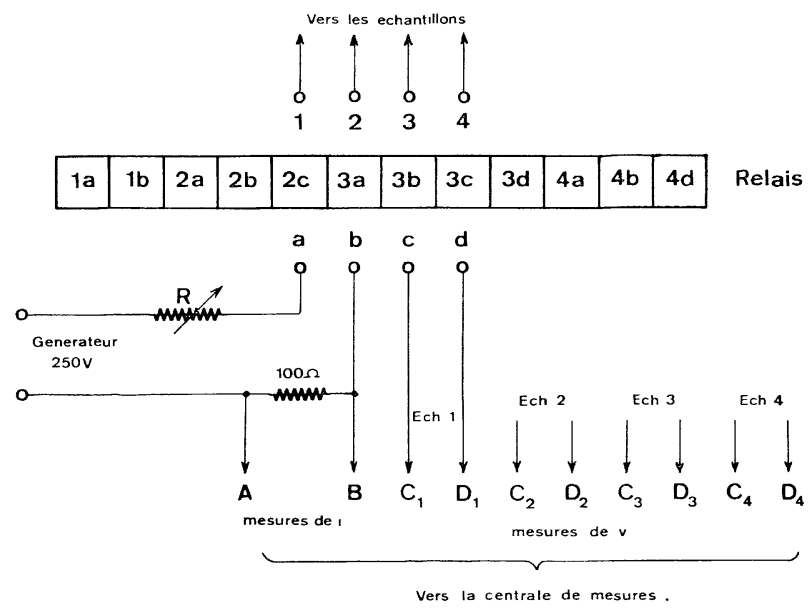

FIG. 2.

3. L'ÉLEGTroaimant possède une alimentation stabilisée, il crée un champ maximum de 7000 gauss lorsque la queue du cryostat est engagée dans l'entrefer.
4. L'ORgane DE commande. - Considérons la figure 2, plaçons-nous dans le cas d'un seul échantillon, le raisonnement reste valable pour les autres échantillons.

Les quatre bornes 1, 2, 3, 4 de l'échantillon doivent être connectées aux bornes a, b, c, d, à l'aide de relais dans l'ordre voulu par le programme de travail du tableau I. Douze relais sont nécessaires pour y parvenir, ils doivent être actionnés chaque fois 4 par 4 .

L'ordre de marche des relais peut être regroupé dans le tableau II.

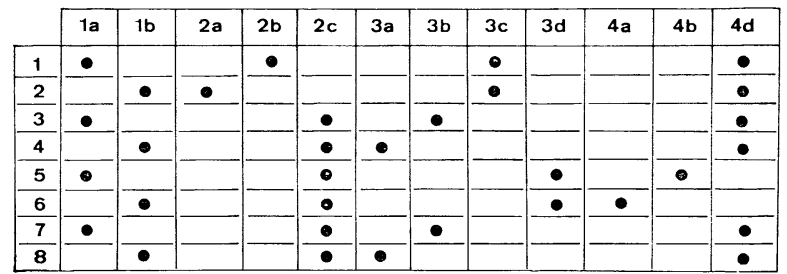

Un petit moteur $\mathrm{M}_{1}$ faisant un tour en $4 \mathrm{mn}$ envoie, en actionnant des contacteurs, des tops de commande aux différents organes (fig. 3). Il s'arrête à la fin de chaque tour.

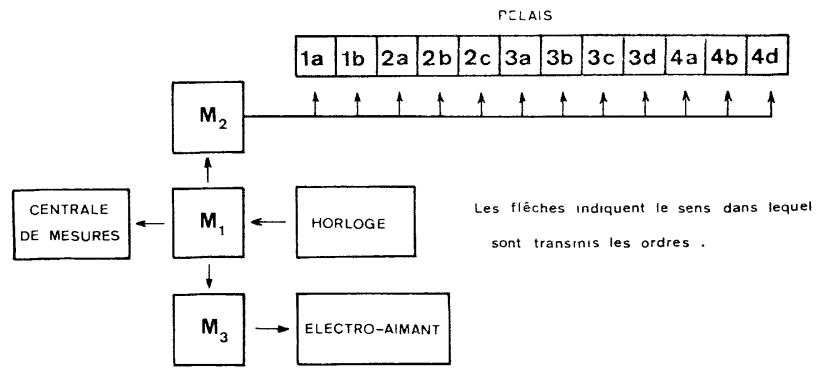

FIG. 3.

- Une série de 16 tops, un toutes les $15 \mathrm{~s}$, déclenchent un moteur $\mathrm{M}_{2}$ fonctionnant pas à pas $(1 / 16$ de tour par pas) qui entraîne un tambour à cames agissant sur des microrupteurs de commande des 12 relais. Ainsi le programme de travail du tableau I est-il exécuté ligne par ligne, à raison d'une ligne toutes les $15 \mathrm{~s}$. - Deux tops, produits après la ligne 6 et après la ligne 8 du programme, provoquent, le premier la mise en route de l'électroaimant, le second l'arrêt de l'électroaimant. Le moteur $\mathrm{M}_{3}$ est chargé d'actionner les interrupteurs de marche et d'arrêt de l'électroaimant.

- Les démarrages de la centrale de mesures ont lieu immédiatement après l'enclenchement des relais, le moteur $\mathbf{M}_{1}$ se charge de la synchronisation.

- Une horloge ordonne le démarrage du moteur $M_{1}$ et par conséquent du double cycle de mesure à la cadence désirée par l'utilisateur.

5. LA GeNTRALE DE mesure est en permanence branchée sur les deux thermocouples et aux bornes $\mathrm{A}$, $\mathrm{B}, \mathrm{C}_{1}, \mathrm{D}_{1}, \mathrm{C}_{2}, \mathrm{D}_{2}, \mathrm{C}_{3}, \mathrm{D}_{3}, \mathrm{C}_{4}, \mathrm{D}_{4}$ de la figure 2 . 
Elle scrute successivement à la cadence de 1 par seconde les sept tensions correspondant aux valeurs de l'une des lignes du tableau I. Les lectures s'inscrivent sur le papier d'une machine à écrire et éventuellement sous forme de cartes perforées. Chaque série de mesure est séparée de la suivante par l'inscription automatique de l'heure, évitant ainsi toute confusion. Les cartes perforées permettent d'atteindre sans perte de temps les valeurs de $\rho$ et de $R_{\mathrm{H}}$ en soumettant à un ordinateur les calculs exprimés par les équations (2) et (4).
V. Conclusion. - L'appareil de mesure de résistivité et de constante de Hall sur des échantillons semiconducteurs s'est révélé très utile dans le Laboratoire depuis sa réalisation.

Il évite aux chercheurs de consacrer leur temps à un travail de routine, il ne présente aucune difficulté d'emploi et sa mise en route est instantanée.

La rapidité de fonctionnement n'a été limitée que par la vitesse de frappe de la machine à écrire ou de la perforatrice.

\section{BIBLIOGRAPHIE}

[1] VAN DER PAUW (L. J.), Revue technique Philips, 1958-59, 20, 234. 